\title{
Hidden Tools. The Use Of \\ Architecture As Reference in the Creative Process of Design
}

ALESSANDRA COMO — LUISA SMERAGLIUOLO PERROTTA University of Salerno 
The topic of the use of architecture as reference within the creative architectural design process is not a matter given for granted, both in the architectural culture and in the teaching.

The Italian architecture studio courses are based on theoretical lectures and laboratory activities. The student's design experience is built through the combined action of theory and practice. The theoretical lectures offer the opportunity to address design issues also through discussion of examples of architecture. What are the examples of architecture that we can consider useful for the design project? And how do students use them within the design project?

This paper aims to answer the questions through a theoretical investigation and through the presentation of an architectural design studio at the University of Salerno (Italy). Through the combination of theoretical issues that underlie the topic and the teaching experience, we want to give a contribution on the relationship between the architecture case studies and their use within the design process.

Within the commented architecture studio work, the architecture examples used as a reference become, through the teacher's guide, as fragments to be reinterpreted within the creative process, able to stimulate the student the recognition of architectural issues. The proposed use of architecture as reference cannot be considered a real hidden layer within the teaching because it is not really hidden; it could be considered a hidden tool that activates the design project, at the halfway between theory and practice, as a critical act of reading and interpretation.

Traditionally, in the Italian schools, the design project is developed through intertwined actions that consider a series of issues as the analysis of the site, the functional program, the interpretation of the topic, history development and so on. Along this extent, the architecture used as a reference is central being used analogically or as a technical-constructive model. This methodology leaves little space to the creative work.

On the contrary, the teaching experience here presented, starts from the necessity of combining architectural design issues with a creative moment autonomous within the design process. The study of the architecture as reference becomes a critical act, a thematic reading of the existing example in order to construct a preliminary reference to the project and more generally it aims at the construction of a design imaginary for the students composed of a set of fragments of spatial solutions, with measures and systems of relation.

The use of the fragment as a creative tool within the project follows a long tradition in the Italian architecture that unites classical and contemporary worlds, from Piranesi to Aldo 
Rossi, the visual research of the avant-garde of the 70 s and the montage-collage of a group of Italian designers in these years.

The example of architecture is therefore not only studied as an evolutionary historical step of the architectural design thinking but as an experience of modelling space, studied outside the historical dimension in order to highlight its main design issues.

The aim of this paper is to show the use of architecture as reference in the creative process of design as a teaching tool for the construction of an imaginary world of forms and spaces for the students and also as an opportunity to develop critical position within the architectural process. At a time when the world is dominated by images and the channels of knowledge are closer, critical thinking thus becomes an opportunity to filter the references and to shift from the complexity of the built architecture to the understanding of space as the main key of the architectural design.

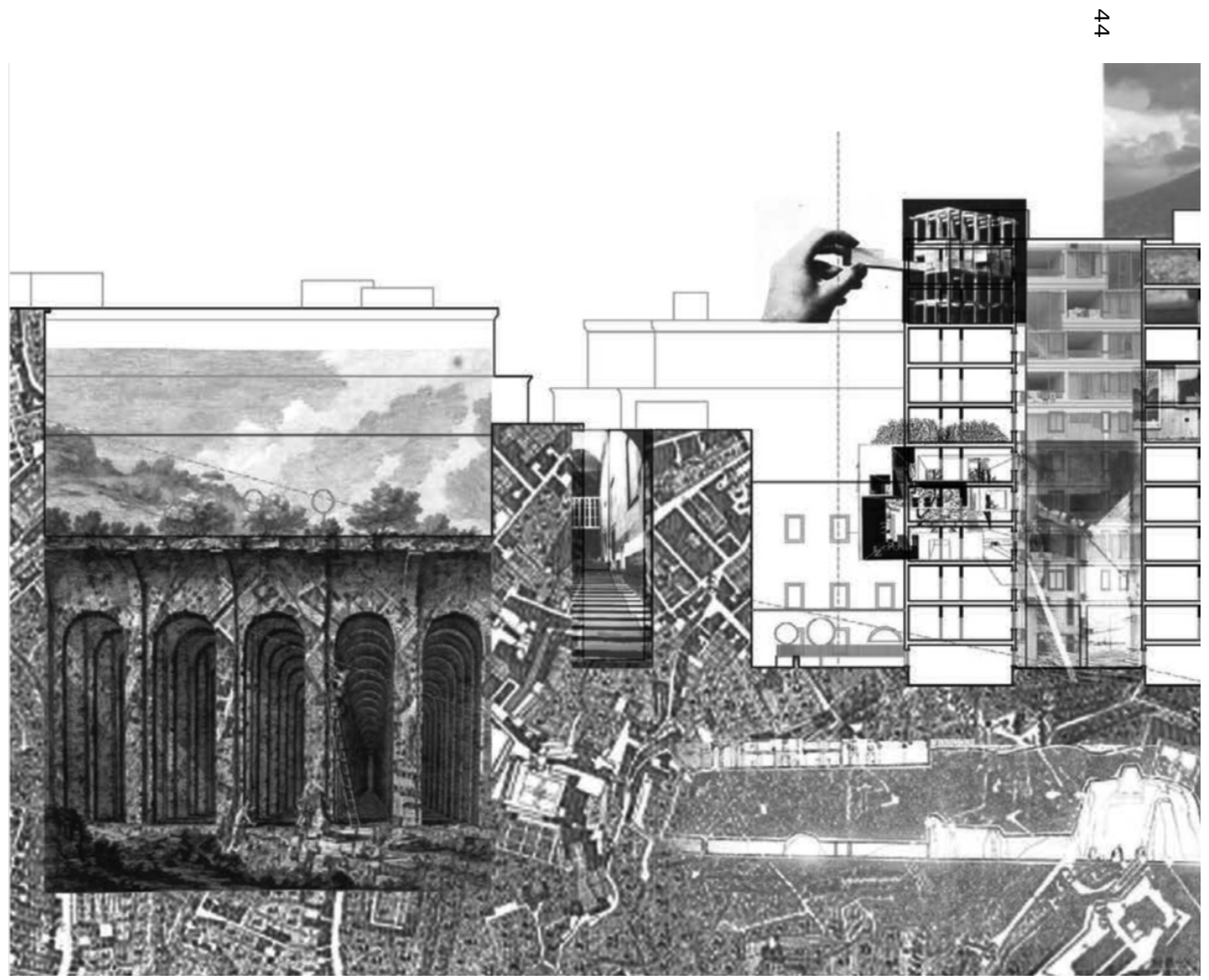




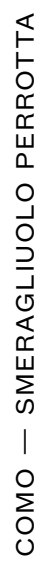

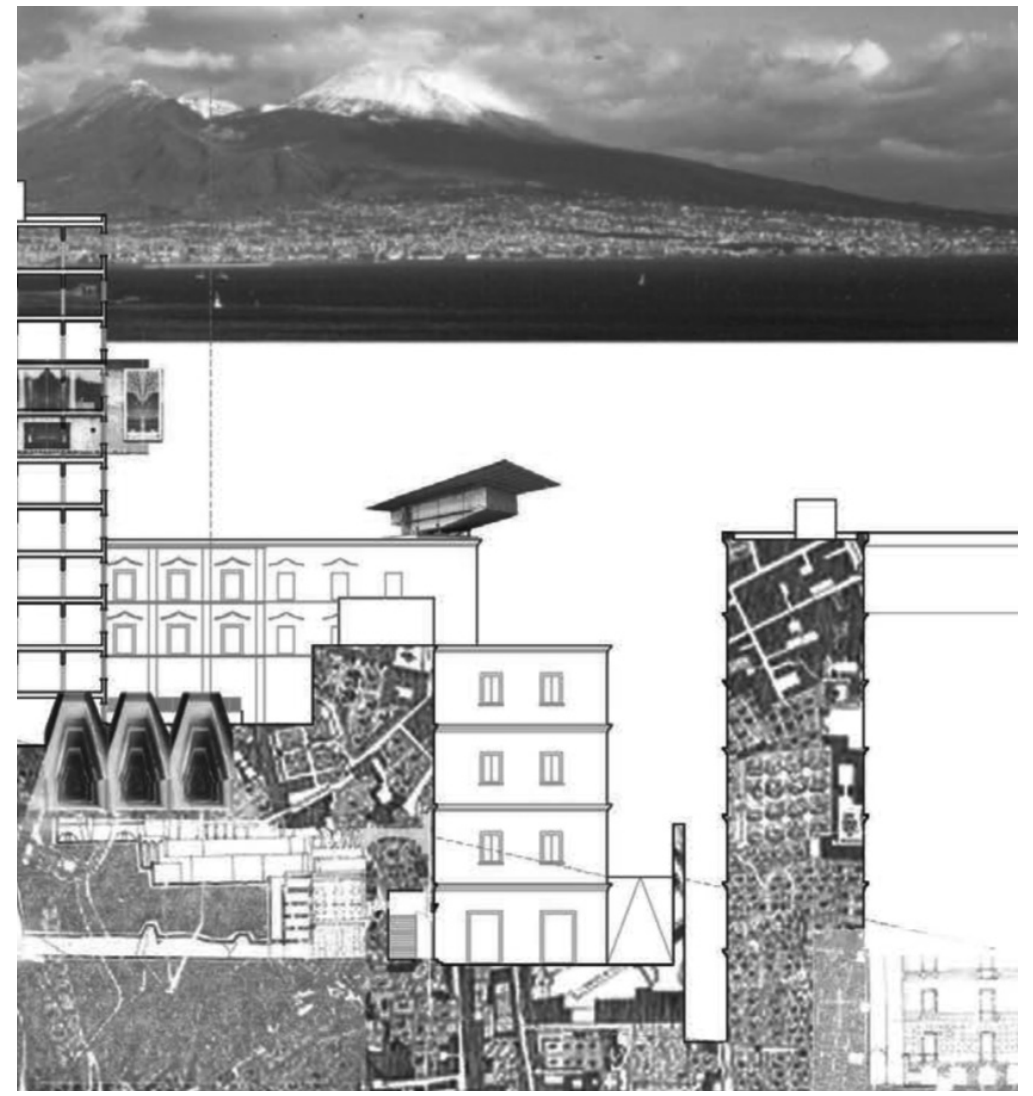

